\title{
Save Here: Emergency Response Application
}

\author{
Siddharth Rai ${ }^{1}$, Hemangi Taraviya ${ }^{2}$, Dipti Pawade ${ }^{3}$, Ashwini Dalvi ${ }^{4}$, Irfan Siddavatam ${ }^{5}$ \\ \{siddharth.rai@somaiya.edu' ${ }^{1}$, hemangi.taraviya@somaiya.edu ${ }^{2}$,diptipawade@somaiya.edu ${ }^{3}$ \} \\ ${ }^{1,2,3,4,5}$ Department of Information Technology, K J Somaiya College of Engineering, Vidyavihar, \\ Mumbai, India
}

\begin{abstract}
The current ratio of medical emergency services available in India is very limited in comparison to the actual population of our country India. Due to this, nearly $27 \%$ of the total deaths in India happen because of the absence of medical attention at the time of medical emergency. In this paper, we have proposed an application which provides quick medical emergency assistance for a patient, based on the patient's real time location. The allocation of the closest Ambulance driver/Save Here Members and closest Hospital for the patient is also done based on the real time location of the patient, ambulance and Save Here Members. With just a click of a button present on our application, the patient can place a request for medical emergency assistance and then the allocated nearest Ambulance driver / Save Here Member would be notified about the patient's details and location. The allocated Ambulance/Save Here Member providing medical emergency assistance to the patient, is shown the shortest route to the patients pick up location and then to the closest hospital with the help of our application.
\end{abstract}

Keywords:Medical Emergency Assistance, Live Location tracking, Shortest route calculation, Euclidian Distance, A* Algorithm

\section{Introduction}

According to the survey carried out by the Indian government, it was found that around $33 \%$ road accidents and $99 \%$ of cardiac arrests victims do not survive due to lack of access to quality treatment during the "golden hour" [1]. The Government's emergency response system takes nearly $44.5 \mathrm{~min}$ for an ambulance to reach hospital in case of a medical emergency. This time is unacceptable as the optimum time to reach the hospital should not exceed more than 30 minutes, according to international guidelines. The government data also shows that every second victim of a heart attack in India takes more than 400 minutes to reach a hospital, which is almost 13 times more than the ideal window of 30 minutes. In the current system, when an individual needs emergency medical assistance, that individual must contact a particular hospital, and only then that hospital arranges an ambulance, which starts from the hospital and rushes to the patient's location in order to get the patient to the hospital, factors like distance and traffic might increase the time taken by the ambulance to complete its duty. In case of emergencies, time plays a very crucial role. Therefore, the main purpose of our paper is to develop an android application which ensures that the patient reaches the hospital in the shortest time possible. So we developed a medical emergency response application called Save Here Emergency Response Application. Using the above mentioned android application, a patient can make a request for medical emergency assistance to the closest 
Ambulance/ Save Here Members based on the real time location of the patient, ambulance and Save Here Members. The Save Here Members is a community created through our application which consists of all the users of our application 'Save Here Emergency Response Application' and these members provide medical emergency assistance to a patient when needed. Using Euclidean Distance with the help of using real time location of the patient, ambulances and Save Here Members we send notification requests to the nearby ambulance drivers and save here members to provide medical assistance to the patient and based on the acceptance of the notification request, the application allocates an Ambulance / Save Here member for the patient. A* Algorithm is used for calculating the shortest and fastest path to the patient and closest hospital ,so that the allocated Ambulance Driver / Save Here Member can pick up and drop the patient to the closest hospital in the shortest time possible.

\section{Literature Survey}

In the research paper titled 'Ambulance Assistance for Emergency Services' [2], a Geo Positioning System was installed in all the ambulances in a particular hospital such that the hospital management system can live track all of its ambulances and assign the ambulance in case of a medical emergency The above method was adopted by the hospital in order to save time and ensure that the patient reaches the hospital as early as possible. The main drawback of this project is that the patient must contact the particular hospital in case of an emergency and it is the responsibility of that hospital to manually assign an ambulance and manually direct the ambulance to the patient's pick up location and bring him to the hospital for medical emergency treatment. The idea of finding the real time position of the ambulance is one of the motivations for our application. In the research paper titled 'Mobile-Based Medical Emergency system' [3], it explores the problems faced by patients living in rural areas of a village in South Africa, when finding an ambulance during a medical emergency. Due to poor communication and poor road network, an ambulance is unable to reach the patient on time, which leads to the death of the patient. The Mobile-Based Medical Emergency Ambulance Scheduling System aims to provide ambulance facility using location-based services in order to avoid death of the patient due to unavailability of an ambulance. From the above discussed paper, it provided us the motivation to provide accurate real time communications between patient and medical forces in our application along with the use of location based services. In the paper titled 'Lisa-life saver' [4], LISA a web application system is used to share the accident location information to nearby ambulances, police stations and to the casualty peer group members. Tracking of the real time location of an ambulance is very important during a medical emergency. In the paper titled 'Ambulance Tracking System Using Restful Api [5],navigator.gelocation method was used on Rest FUL web services which updates ambulance location on the database and the location is taken from the database. Due to the live location tracking of ambulances, the patient is well informed of the live location of the ambulance. TAZ_OPT: A goal programming model for ambulance location and allocation[6], determines the satellite location of the ambulances. It is a programming model, developed to facilitate the decision making process when dispatching an ambulance. In paper titled 'Role of Parallelism in Ambulance Dispatching'[7], for allotting a particular ambulance in a particular zone, it considers idle as well as busy units. The parallelism, applied upon the centrality policy, results in timely arrival of ambulance. In the paper titled 'Modeling Metropolitan-Area Ambulance Mobility Under Blue Light Conditions' M [8], it proposed an 
approach which leads to improvements in ambulance operational efficiency through the automated generation of more effective response strategies and tactics. This approach uses a large historical dataset of incidents and ambulance location traces to model route selection and arrival times. This is an attempt to apply a data-driven methodology for route selection and the estimation of arrival times of ambulances travelling with blue lights and sirens on. Automatic Ambulance Rescue With Intelligent Traffic Light System[9] consists of autonomous collecting; integrating and sharing EMS based on personal profiles enhances emergency activities of paramedics on emergency scenes and clinicians in ER, resulting in increased survival rate.

According to the Survey, we found that most of the paper focuses mainly on tracking of the Ambulances and not the Allocation of the Ambulance. Allocation of the ambulance on time and providing the ambulance driver with the shortest and the fastest route, may save the life of the patient. Since there is an increase in demand for quick medical emergency assistance our paper focuses on both real time location tracking of the ambulance/Save Here Members and allocation of the closest Ambulance/Save Here Members and closest Hospital based on the real time location of the patient. This application ensures that the patient is dropped to the hospital in the shortest time possible.

\section{Methodology}

The most crucial issue in a medical emergency service is the time factor. So, we propose a system in which the medical emergency service can take place in the shortest time possible.The medical emergency service is achieved with help of the ambulance drivers and Save Here Members. The Save Here Members is a community created through our application which consists of all the users of our application 'Save Here Emergency Response Application' and these members provide medical emergency assistance to a patient when needed. Fig. 1 gives the implementation overview of the proposed system. The system is divided into three functional modules viz. 1. Location Fetching and 2. Ambulance and Save Here Member allotment and 3. Finding the shortest route and location tracking.

\subsection{Location Fetching}

First the live location of the patient is fetched when the patient [user] places a request for an emergency medical assistance. The real time location is found by fetching the live location coordinates with respect to the longitude and latitude of the smartphone on which the request for medical emergency assistance was placed through our application. 


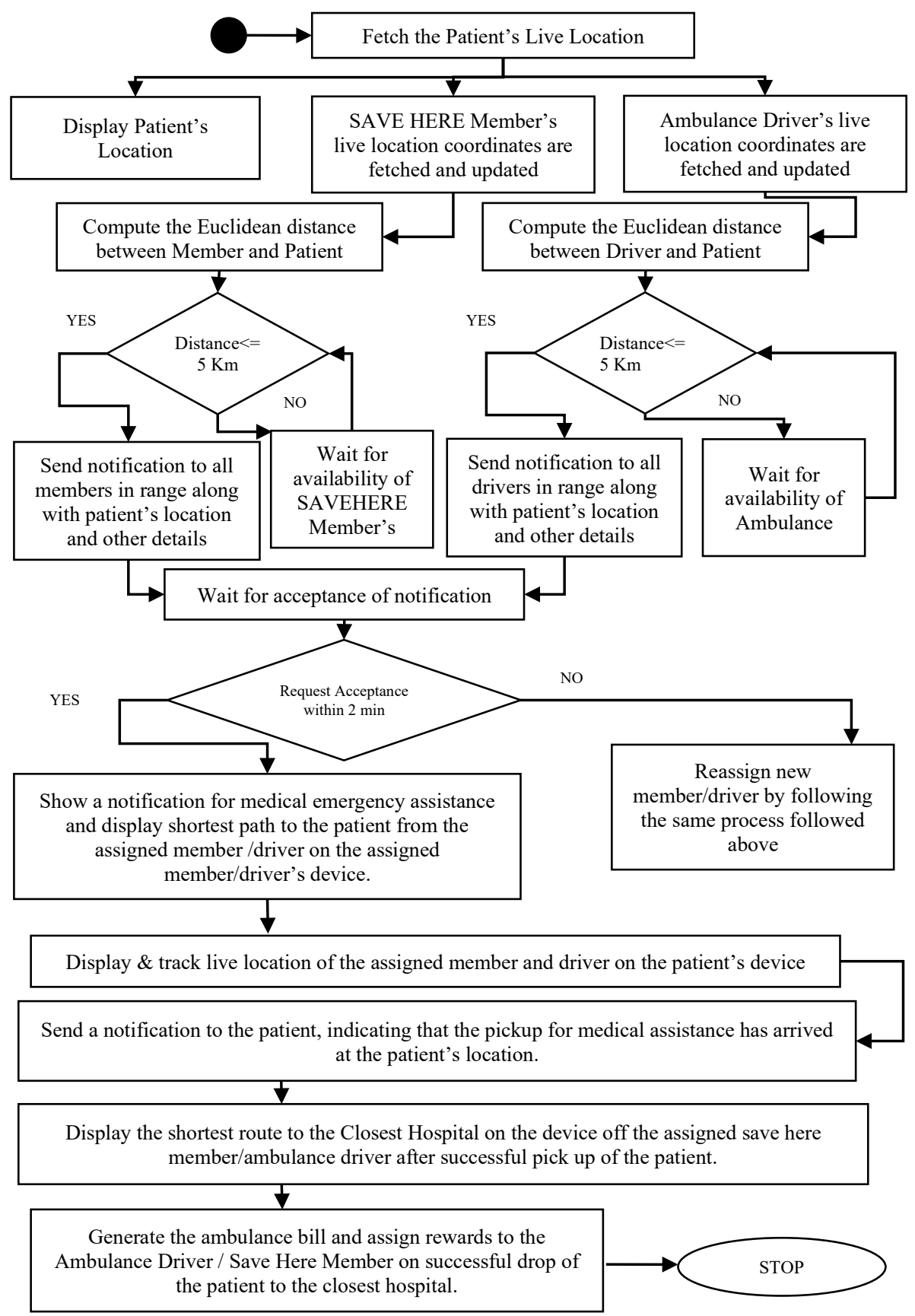

Fig. 1. Implementation Flowchart

This live location coordinates is stored in the Google Firebase (database) and its value is updated frequently. After successfully fetching the live location of the patient, his/her live location will be shown on the Google Maps present in our application using the Google Maps 
API [10]. In the same way, the live location coordinates of each and every registered Ambulance Drivers and Save-Here Members is fetched and updated frequently and is stored in the Google Firebase.

\subsection{Ambulance and Save Here Member allotment}

After fetching the live location coordinates, the distance between the patient and all the ambulance drivers /Save Here Members is calculated. The distance is calculated using raw Euclidean method [11] given in equation 1, where the coordinates i.e. longitude and latitude of the patient and ambulance drivers /Save Here Members act as inputs.

Euclidean Distance $=\sqrt{ }\left((\mathrm{x} 2-\mathrm{x} 1)^{2}+(\mathrm{y} 2-\mathrm{y} 1)^{2}\right)$

Now after finding the distance, the Ambulance Drivers /Save Here Members which are within the radius of five kilometers from the patient are filtered out. Then an alert containing medical emergency assistance request is sent to the filtered out Ambulance Drivers /Save Here Members which are within the radius of five kilometers from the patient. The Ambulance Driver and Save Here member who accepts the request first, gets allotted to the patient who places a request for medical emergency assistance.

\subsection{Finding the shortest route and location tracking}

Once the medical emergency assistance request is accepted then the shortest path fromthe Ambulance Driver's and Save Here Member's location to the patient's location is generated using the $A^{*}$ algorithm. The reason why we are using $A^{*}$ algorithm over Dijkstra algorithm is that Dijkstra algorithm is an exhaustive search algorithm i.e. Dijkstra has to explore in all directions whereas $A^{*}$ can explore towards the goal [12-13]. First of all over here, the path present on the map is converted into a graph format showing the Start and End location.The intersection on the map is as well as the starting and the end pointsare considered as nodes of the graph created[14][15]. The roads on the map are considered as the edges of the graph.Conversion of the actual map to a graph also depends on the level of hierarchy of the map conversion. Then $\mathrm{A}^{*}$ algorithm given by equation 2 is applied.

$f(n)=g(n)+h(n)$

Where $n=$ next node on the path, $f(n)=$ Evaluation Function , $g(n)=$ distance from start node to $\mathrm{n}$ and $\mathrm{h}(\mathrm{n})=$ heuristic function i.e. distance from $\mathrm{n}$ to end node.

To calculate the heuristic function, initially we have considered two methods viz Euclidean distance method and Manhattan distance. But then continued using the Euclidean method instead of Manhattan distance since Manhattan distance generally works only if the points are arranged in the form of a grid and the problem which we are working on gives more priority to the distance between the points on a map in which grid form might not exist in every scenario.

For example:-

Vertex coordinate 1 is 287,424 i.e. $\mathrm{x} 1=19.16453$ and $\mathrm{y} 1=72.93767$

Vertex coordinate 2 is 669,648 i.e. $\mathrm{x} 2=19.21247$ and $\mathrm{y} 2=72.97678$

Euclidean Distance $=\sqrt{ }(\mathrm{x} 2-\mathrm{x} 1)^{2}+(\mathrm{y} 2-\mathrm{y} 1)^{2}=\sqrt{ }(0.04794)^{2}+(0.03911)^{2}=0.061869 \mathrm{~km}$

Manhattan Distance $=|\mathrm{x} 1-\mathrm{x} 2|+|\mathrm{y} 1-\mathrm{y} 2|=|\mathrm{x} 1-\mathrm{x} 2|+|\mathrm{y} 1-\mathrm{y} 2|=0.08705 \mathrm{~km}$

From the above observation it is understood that Euclidean distance gives the shortest distance compared to Manhattan distance, Therefore we use Euclidean distance to calculate 
heuristic function $h(n) . g(n)$ which is the distance from the start node to the current node ' $n$ ' is the actual distance in $\mathrm{km}$ between the two nodes. After getting $\mathrm{g}(\mathrm{n})$ value and $\mathrm{h}(\mathrm{n})$ for each and every edge and node we can perform $\mathrm{A}^{*}$ algorithm, which will provide us with the shortest path form the Ambulance driver/ Save Here Members who have accepted the medical emergency assistance request to the live location of the patient.Once the Ambulance driver/ Save Here Members reaches the location and successfully picks up the patient who needs medical emergency assistance, then again $\mathrm{A}^{*}$ algorithm is implemented by our application to provide the shortest path to the closest hospital.Once the Patient is successfully dropped to the hospital, a cost report is generated automatically and sent to the patient while simultaneously reward points are allotted to the ambulance driver andSave Here Members who helped the patient in providing medical emergency assistance successfully. Note if the ambulance driver / Save Here Members helping the patient are not able to drop the patient to the nearest hospital, then a new emergency request is placed, so that other Ambulance Driver / Save Here Members can come and save the patient and the same process mentioned above continues again. Throughout the process the location of ambulance as well as the Save Here member can be tracked using the Google Maps present in our application using the Google Maps API [10].

\section{Results}

The main objective of this application is to allot an ambulance /Save Here Member, which is at a closest distance from the location of the patient requesting medical emergency assistance and reduce the time required to provide the medical emergency assistance based on the current live locations of the users(i.e. patients, ambulance driver and Save Here Members) stored in Google Firebase.

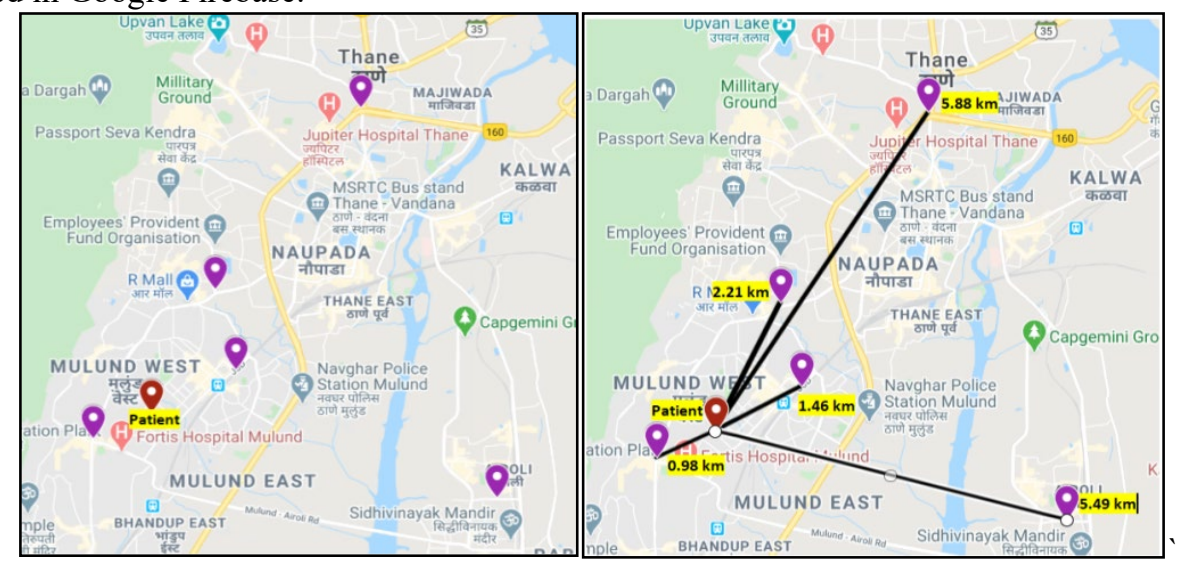

(2)

Fig. 2. Live location coordinates of the patient, ambulance driver and Save Here Members Fig.3. Euclidean distance between patient and ambulance driver/ Save Here Members 


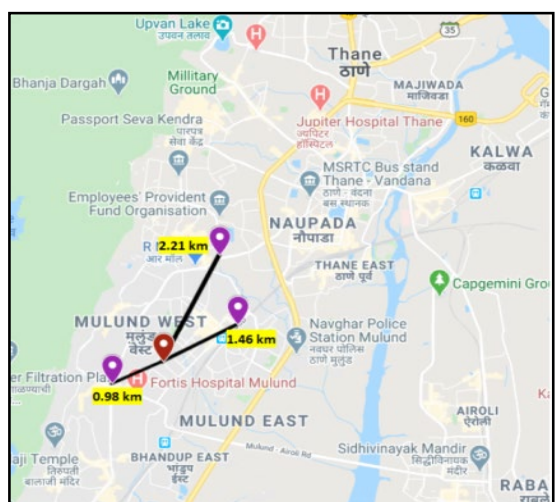

(4)

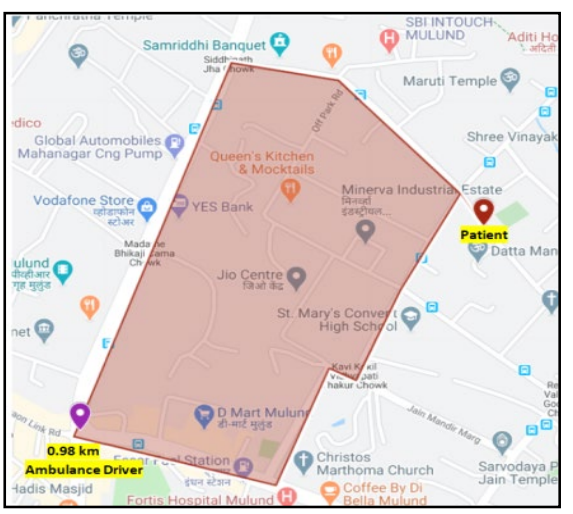

(5)

Fig.4. Ambulance drivers / Members within radius 5 Km Fig. 5. Map to graph Conversion

As shown in figure 2 the live location coordinates of the patient, ambulance driver and Save Here Members can be seen on the map. Next task is to calculate the distance between the patient and ambulance /as the Save Here Members which is shown in figure 3.

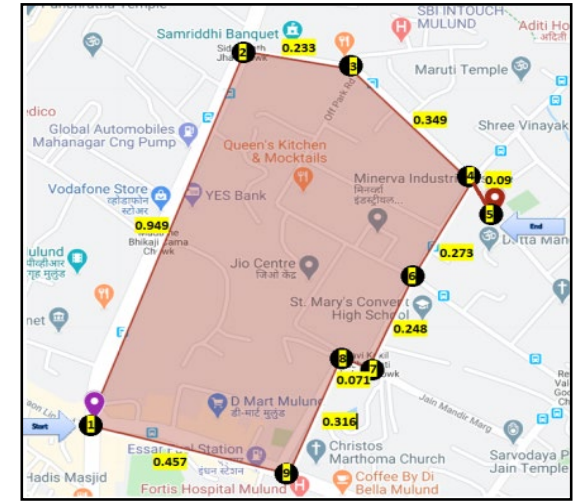

(6)

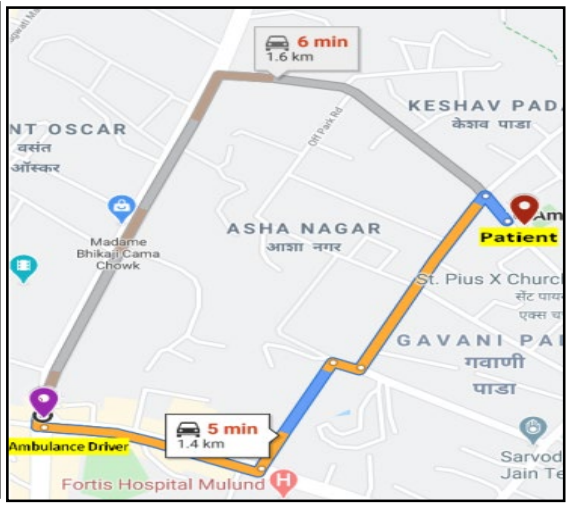

(7)

Fig. 6. Graph with nodes and distance

Fig. 7. Shortest Route from Ambulance to Patient

After the first ambulance driver/ Save Here Member within the $5 \mathrm{~km}$ radius from tha patient's location accepts the request for medical emergency assistance for that patient, then the shortest path to the patient is calculated using $A^{*}$ Algorithm from the ambulance driver/ Save Here Member location who accepted the request to provide assistance. $g(n)$ is the actual distance in kilometers from the start node to the current node. Heuristic Function $[\mathrm{h}(\mathrm{n})]$ which is the Euclidean distance between each node to the end node is calculated. Table 1 gives the heuristic function for each node and its associated distance to the end node.Obtaining $\mathrm{g}(\mathrm{n})$ and $\mathrm{h}(\mathrm{n})$ valuesfor each and every edge and node we can perform $\mathrm{A}^{*}$ algorithm, which will provide the shortest path form the Ambulance driver/ Save Here Members to the patient. Therefore as per the statistics given in table 2, the shortest and fastest route is route 1-9-8-7-64-5 found using $A^{*}$ algorithm is displayed on the drivers smart phone through our application just as the image shown in figure 7. 
Table 1: Heuristic function value for each node

\begin{tabular}{|c|c|c|c|c|c|}
\hline $\begin{array}{c}\text { Heuristic } \\
\text { Function }\end{array}$ & Distance $(\mathrm{km})$ & $\begin{array}{c}\text { Heuristic } \\
\text { Function }\end{array}$ & $\begin{array}{c}\text { Distance } \\
(\mathrm{km})\end{array}$ & $\begin{array}{c}\text { Heuristic } \\
\text { Function }\end{array}$ & $\begin{array}{c}\text { Distance } \\
(\mathrm{km})\end{array}$ \\
\hline $\mathrm{h}(1)$ & 1.01 & $\mathrm{~h}(4)$ & 0.091 & $\mathrm{~h}(7)$ & 0.460 \\
\hline $\mathrm{h}(2)$ & 0.678 & $\mathrm{~h}(5)$ & 0 & $\mathrm{~h}(8)$ & 0.485 \\
\hline $\mathrm{h}(3)$ & 0.464 & $\mathrm{~h}(6)$ & 0.234 & $\mathrm{~h}(9)$ & 0.771 \\
\hline
\end{tabular}

Table 2: Shortest route calculations

\begin{tabular}{|c|c|c|c|c|c|}
\hline Start & Destination & Route & $\begin{array}{c}\text { Distance } \\
(\mathrm{Km})\end{array}$ & $\begin{array}{c}\text { Time (Considering } \\
\text { constant average Speed) } \\
(\mathrm{min})\end{array}$ & $\begin{array}{c}\text { Shortest } \\
\text { Path }\end{array}$ \\
\hline 1 & 5 & $1-2-3-4-5$ & 1.6 & 6 & No \\
\hline 1 & 5 & $1-9-8-7-6-4-5$ & 1.4 & 5 & Yes \\
\hline
\end{tabular}

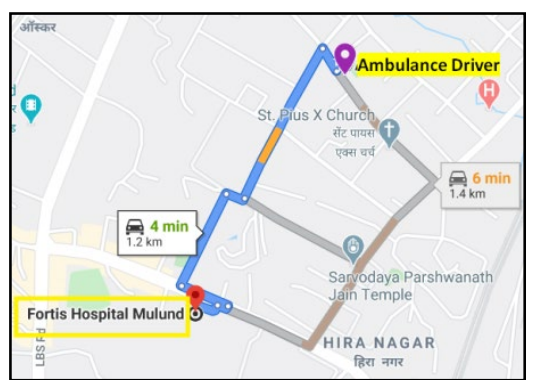

Fig. 8. Shortest Route from Ambulance driver to the nearest hospital

Both the patient and allotted ambulance driver/save here member can see each other's Live Location updated frequently, on the Google Maps present in our application using the Google Maps API. Once the ambulance drivers / save here member reaches the location of the patient and picks the patient up, again a shortest path is computed from the ambulance driver/save here members location to nearest hospital above using again the same method of $\mathrm{A}^{*}$ algorithm as above. Figure 8 gives the shortest route that is displayed on ambulance driver's/ save here members smart phone through our application.

\section{Conclusion}

Save Here: Emergency Response Application ensures to provide medical emergency assistance in the shortest time possible. It is developed in order to reduce the fatality rate of patients when quick and fast ambulance services are not available. Quick allocation of medical forces and providing the shortest and fastest route in order to reach the patient and closest hospital in an emergency is the main goal of our application. The medical emergency service is achieved with help of the ambulance drivers and Save Here Members. Thus if implemented in densely populated countries like India, our application can produce better results.

\section{References}

[1] https://www.downtoearth.org.in/coverage/ambulance-emergency-40605 Access on: June 19

[2] Shantanu S.,"Ambulance Assistance for Emergency Services Using GPS Navigation”, International Research Journal of Engineering and Technology, Vol 03(09), Sep-2016, Pp. 1328-1331. 
[3] Bassey Isong, Nosipho Dladlu, Tsholofelo Magogodi,"Mobile-Based Medical Emergency Ambulance Scheduling System", International Journal of Computer Network and Information Security(IJCNIS), Vol.8, No.11, 2016, pp.14-22.

[4] P. Iyappan, B. NanthiniDevi, P. Nivedha and V. Sayoojya, "Lisa-life saver," 2019 IEEE International Conference on System, Computation, Automation and Networking (ICSCAN), Pondicherry, India, 2019, pp. 1-6.

[5] C. S. Vikas, Immanuel A. Ambulance Tracking System Using Restful Api. Orient.J. Comp. Sci. and Technol;10(1)

[6] A. Shuib and Z. A. Zaharudin, "TAZ_OPT: A goal programming model for ambulance location and allocation," 2011 IEEE Colloquium on Humanities, Science and Engineering, Penang, 2011, pp. $945-950$

[7] S. Lee, "Role of Parallelism in Ambulance Dispatching," in IEEE Transactions on Systems, Man, and Cybernetics: Systems, vol. 44, no. 8, pp. 1113-1122, Aug. 2014.

[8] M. Poulton, A. Noulas, D. Weston , G. Roussos, "Modeling Metropolitan-Area Ambulance Mobility Under Blue Light Conditions," in IEEE Access, vol. 7, pp. 1390-1403, 2019

[9] Sangeetha, K.. (2014). Automatic Ambulance Rescue With Intelligent Traffic Light System. IOSR Journal of Engineering. 4. 10.9790/3021-04255357.

[10] https://developers.google.com/maps/documentation Access on: Aug 2019

[11] Daniel N., Schmitz G., Carolinne G., Tássia A., Marcelino da Silva, "Analysis of the difference between the Euclidean distance and theactual road distance in Brazil", 17th Meeting of the EURO Working Group on Transportation, 2-4 July 2014,Sevilla, Spain

[12] Madkour, Amgad Aref, Walid \& Rehman, Faizan \& Rahman, Abdur \& Basalamah, Saleh. (2017). A Survey of Shortest-Path Algorithms, arXiv:1705.02044v1 [cs.DS] 4 May 2017

[13] Harika Reddy, "PATH FINDING - Dijkstra's and A* Algorithm's" December 13, 2013 Downloaded from: http://cs.indstate.edu/hgopireddy/algor.pdf

[14] K. Vijayakumar, Chokkalingam Arun, "Integrated cloud-based risk assessment model for continuous integration",Int. J. Reasoning-based Intelligent Systems", Vol. 10, Nos. 3/4, 2018.

[15] K. Vijayakumar, S. Suchitra and P. Swathi Shri, "A secured cloud storage auditing with empirical outsourcing of key updates”, Int. J. Reasoning-based Intelligent Systems, Vol. 11, No. 2, 2019. 